\title{
Escolha de destino turístico: Estudo bibliométrico com análise de citação e co-citação de autores
}

\author{
Claudio Sunao Saito ${ }^{1}$ e Vivian Iara Strehlau \\ Escola Superior de Propaganda e Marketing - ESPM, São Paulo, SP, Brasil
}

\section{DETALHES DO ARTIGO}

\section{Histórico do Artigo:}

Recebido: 12 de Dezembro de 2016

Revisado: 26 de Junho de 2017

Aceito: 06 de Outubro de 2017

Disponível online: 12 de Novembro de 2017

Sistema de revisão "Double blind review"

Editor Científico

Renata Galhone

\section{Palavras-chaves:}

Turismo;

Escolha de destino;

Processo de decisão;

Bibliometria;

Análise de citação.

\section{RESUMO}

O estudo bibliométrico foi realizado com o objetivo de entender os principais assuntos, abordagens e referenciais teóricos relacionados ao tema "escolha de destino turístico". A pesquisa, realizada a partir da base de dados Scopus e com utilização de softwares estatísticos, foi baseada na técnica de análise de citação, definindo-se a relevância e prestígio dos artigos, autores, modelos e fundamentos teóricos com base no número de citações realizadas em artigos posteriores. Os resultados da pesquisa demonstram que existe diversidade de perspectivas e abordagens relacionadas ao tema e que os artigos podem ser agrupados em estudos relativos a modelos de decisão ou escolha de destino, fatores motivadores para turismo, características ou fatores pessoais, características e atrativos do destino, natureza da viagem pretendida (tamanho do feriado, distância, duração da viagem, etc.), experiências da viagem (humor, sentimentos durante a viagem, avaliações pós-viagem, etc.), influência da imagem do destino e influência da informação/comunicação na escolha do destino turístico. Os resultados também indicam uma pulverização em relação à origem dos artigos (países/instituição), autores e referenciais teóricos utilizados em pesquisas relacionados ao tema.

(C) 2018 Internext | ESPM. Todos os direitos reservados!

\section{INTRODUÇÃO}

Nas últimas seis décadas, o turismo internacional se tornou um dos setores da economia com maior e mais rápido crescimento em todo o mundo. Segundo o World Tourism Organization - UNWTO (2015), o número de turistas internacionais que chegam a países em todo o mundo (e que pernoitam no destino) cresceu de 25 milhões em 1950 para 1.133 bilhões em 2014, enquanto a receita com turismo internacional atingiu o valor de US\$ 1.245 trilhões em todo o mundo. A previsão de crescimento de turistas internacionais em todo o mundo todo é de 3,3\% ao ano, chegando a 1.8 trilhões em 2030.

Com crescimento do setor de turismo no mundo e do aumento da sua relevância na economia de vários países, o número de pesquisas acadêmicas relacionadas ao assunto aumentou nas últimas décadas, inclusive na área de ciências sociais.

\footnotetext{
${ }^{1}$ Contato do autor - Email: csaito@espm.br

Segundo Sampaio (2013), tendo o turismo se tornado uma prática social alargada a partir da segunda metade do século XX, figura hoje dentro das ciências sociais como um tema de vasto número de conferências e publicações especializadas, ancoradas nas mais diversas perspectivas. Na Administração uma área aplicada dentro das ciências sociais também se verifica grande interesse pelo assunto "turismo", principalmente em estudos ligados ao comportamento do consumidor. Correia e Pimpão (2008) argumentam que, com o desenvolvimento global do turismo, o entendimento do comportamento do consumidor é de fundamental importância, pois a gestão estratégica dos destinos turísticos depende do desenvolvimento de teorias sobre o comportamento do consumidor e entendimento das escolhas dos turistas.

A escolha de destino ou processo de escolha é um tema frequente no estudo do comportamento do 
consumidor em turismo, pois, está relacionado à criação e manutenção da demanda relacionada aos destinos e serviços turísticos oferecidos. Dada a importância do tema, esse estudo bibliográfico foi realizado com o objetivo de identificar os principais assuntos e autores ligados ao tema e, ao mesmo tempo, entender as principais discussões e relações estabelecidas entre eles. Para isso, foram buscados artigos na base de dados Scopus, que foram selecionados através da ferramenta estatística SciMat (Cobo et al., 2012) e analisados - quantitativa e qualitativamente - para a obtenção dos resultados. $O$ estudo demonstra que, no contexto da escolha de destino, as pesquisas são elaboradas com utilização de abordagens e referências teóricas diversas, além de serem realizadas em escolas e países diversos, fato que demonstra o interesse global do tema escolhido como objeto da pesquisa.

\section{ESCOLHA DE DESTINO EM TURISMO}

A literatura do turismo oferece muitos trabalhos relacionados ao estudo do comportamento do consumidor que descrevem o processo de tomada de decisão dos turistas. Sirakaya e Woodside (2005) avaliaram os principais modelos de tomada de decisão existentes na literatura do turismo e concluíram que a maior parte desses modelos entende o processo de seleção (ou escolha) como um funil, onde os viajantes, baseados em comportamento racional, eliminam as alternativas com base em fatores sócio-psicológicos e não psicológicos. De acordo com o estudo, os fatores que determinam o conjunto de alternativas e escolhas podem ser condensadas em quatro grupos, onde a escolha do destino depende da natureza da interação entre essas variáveis: (1) variáveis internas (atitudes, valores, estilo de vida, imagem, motivação, ciclo de vida, redução de riscos, etc.), (2) variáveis externas (atrativos do destino, família, amigos, cultura, grupos de referência, etc.), (3) a natureza da viagem pretendida (tamanho do feriado, distância, duração da viagem, etc.) e (4) experiências da viagem (humor e sentimentos durante a viagem, avaliações pósviagem, etc.). De forma similar, Jang e Cai (2002) afirmam que, embora não exista uma perspectiva unificada, uma abordagem que efetivamente explica as motivações de viagem dos turistas avalia os fatores que impulsionam (push motives) e atraem (pull motives) turistas para um destino específico. As push motives correspondem às variáveis internas $e$ emocionais que levam turistas potenciais a tomar decisões relativas à viagem, explicando o desejo de viajar e o destino considerado, por exemplo, relaxamento, interação social, busca de conhecimento, reconhecimento social e aventura, enquanto as pull motives estão ligados a aspectos externos, motivações inspiradas pela atratividade de um destino, como, por exemplo, ambiente natural, ambiente social, facilidade de compras, gastronomia e atrações culturais e históricas. Para Karl, Reintinger e Schmude (2015), além das características dos turistas, as restrições relacionadas ao destino também desempenham um papel importante na seleção ou rejeição de destinos durante o processo de decisão. As restrições variam de acordo com o tipo de destino, estando mais relacionadas ao destino do que ao turista. Como exemplo podem ser citadas as restrições financeiras (geralmente ligadas às distâncias e meio de transporte da viagem, pois viagens de longa distância são associadas a altas despesas de viagem), a falta de feriados, a impossibilidade de encontrar um companheiro de viagem adequado, além dos perigos e situação política relacionada ao destino.

Em relação ao processo de escolha, Sirakaya e Woodside (2005) citam duas abordagens predominantes na literatura. A primeira, denominada "abordagem comportamental", tem como origem os modelos gerais de comportamento do consumidor como os apresentados por Engel, Kollat e Blackwell (1973) e Howard e Sheth (1969). Essa abordagem sugere que turistas são motivados por diversos fatores e escolha das diferentes alternativas que podem ir ao encontro de suas necessidades e a ênfase dos modelos recaem sobre o processo de compra, onde o turista é exposto às informações, procura mais informações, avalia as alternativas e, finalmente, escolhe uma das alternativas. A principal proposta dos modelos comportamentais é identificar os estágios da decisão e desenhar esse processo identificando os fatores, internos e externos, que influenciam o processo. Um dos modelos mais reconhecidos dessa abordagem é o modelo proposto por Moutinho (1987), que apresenta um fluxograma dividido em três partes (pré-decisão e processos de decisão, avaliação pós-compra e tomada de decisões futuras) onde descreve o processo percorrido pelo turista e as variáveis influenciadoras das decisões relacionadas à compra e recompra de viagens e destinos de férias. Entre as principais variáveis estão as influências ambientais, fatores pessoais (personalidade, estilo de vida, motivação), atitude, 
influência da família, fatores inibidores e de risco e satisfação/insatisfação com a viagem.

A segunda abordagem é denominada "abordagem de conjunto de escolhas" (choice-set). Apesar de aceitar a maioria dos pressupostos da abordagem comportamental, segue em um caminho diferente, mais simples e prático, ao propor que o turista desenvolve um conjunto inicial de opções de destino e depois elimina alguns desses destinos ao longo do tempo até que a escolha final é determinada. Nessa abordagem, o foco não está no processo de decisão, mas sim nas variáveis psico-comportamentais e na forma como os consumidores passam a ter julgamentos cognitivos e afetivos, intenções e compromissos antes de chegar à decisão final. Para Woodside e Lysonski (1989) variáveis do marketing mix exercem influência externa enquanto as variáveis do turista (experiência no destino anterior, ciclo de vida, renda, idade, estilo de vida e sistema de valores) representam as variáveis internas. Ambos afetam a escolhas de destinos que são categorizados em diferentes conjuntos (considerados, ineptos, inertes e não disponíveis/conscientes) que, junto com associações afetivas relacionadas ao destino, influenciam as preferências, intenção e escolha do destino. Um e Crompton (1990) apresentam um modelo mais simples, onde fatores externos (estímulos significativos, simbólicos e sociais) influenciam a construção cognitiva de um conjunto de destinos potenciais na consciência no turista (awareness set). Esses fatores externos juntamente com fatores internos (características pessoais, motivação, valores e atitudes) influenciam a formação cognitiva de um segundo conjunto de destinos considerados (evoked set) na mente do turista, a partir da qual seleciona e escolhe o destino.

Esses modelos, porém, são criticados por Decrop (1999) e Decrop e Snelders (2004) para quem não existe uma, mas diversas formas de entender o processo de decisão e comportamento das viajantes. Para eles, os modelos tradicionais de Moutinho (1987), Um e Crampton (1991), e Woodside e Lysonski (1989) são baseados em paradigmas positivistas, na racionalidade (limitada) e processamento cognitivo das informações, e falham na tradução da complexidade da vida real e no encaminhamento de questões importantes como o papel das emoções e sentimentos, baixo envolvimento e busca passiva das informações, nostalgia, sonhos, etc. Ele também critica o fato dos modelos considerarem principalmente o processo individual de escolha ao passo que em turismo as decisões dependem do individuo, grupo e do momento. Após análise dos modelos de decisão existentes, Sirakaya e Woodside (2005) parecem concordar com as criticas, sugerindo que em situações reais os modelos de conjunto de escolhas (choice sets) podem servir mais como modelos probabilísticos do que determinísticos e que novos modelos de escolha de destino, mais simples, específicos e aplicáveis, devem ser criados considerando-se as diferenças na natureza e finalidade de cada tipo de viagem, o papel desempenhado pelas emoções no processo de decisão (que muitas vezes não é totalmente racional por se tratar de turismo) e as tomadas de decisão realizadas em grupo.

Considerando o papel das emoções na escolha de destinos turísticos, existe na literatura uma discussão sobre a influência do constructo de self-congruity (proximidade ou distanciamento entre a autoimagem do turista e a imagem por ele atribuída ao destino) como fator motivador. Quanto maior o encontro entre a auto-imagem do turista e a imagem do destino por ele atribuída, maior a atitude favorável em relação ao destino e, consequentemente, maior a possibilidade de que visite o destino. Alguns estudos (Sirgy, 1997; Beerli, Meneses, \& Gil, 2007; Boksberger, Dolnicar, Laesser, \& Randle, 2011; Usakli \& Baloglu, 2011) demonstram a influência da selfcongruity nas decisões relacionadas à escolha de destinos turísticos. Outras pesquisas, também relacionadas à congruência entre o turista e o destino, demonstram que a relação entre as características do turista e seu destino de férias determinam a satisfação (Bekk, Spörrle, \& Kruse, 2016) e fidelidade (Ekinci, Sirakaya-Turk, \& Preciado, 2013) do turista em relação ao destino.

No processo de tomada de decisão, é preciso destacar que nem sempre as decisões são tomadas individualmente, devendo-se avaliar pessoas ou grupos que agem como influenciadores no processo. Stone (2016), por exemplo, afirma que, ao invés de tomar suas próprias decisões, uma parte dos turistas delegam decisões sobre onde ir, o que fazer e onde comer, para pessoas com quem viajam. Na pesquisa realizada, uma média de $25 \%$ das pessoas delegaram as decisões de destino turístico aos companheiros de viagem. Para Shu e Scott (2014) os influenciadores não são apenas pessoas mais próximas ou companheiros de viagem, são as redes sociais, que desempenham importante papel na construção da 
atratividade do destino, gerando impressões positivas ou negativas que influenciam os turistas na escolha dos destinos turísticos.

\section{METODOLOGIA DA PESQUISA}

Na realização do estudo, foram utilizadas técnicas, ferramentas e indicadores bibliométricos. Cronin (2001) afirma que estudos bibliométricos tradicionalmente concentraram esforços no acompanhamento de indicadores altamente visíveis e objetivos de atividade acadêmica: publicações e citações. Os indicadores bibliométricos mais utilizados são os indicadores do desempenho científico de organizações, agências e países com base nas contagens de publicações e citações na literatura científica (Narin \& Hamilton, 1996). Em relação ao método, Wallin (2005) afirma que estudos bibliométricos incluem padrões de publicação de estudos, bibliographing (contagem do número de resumos e indexadores bibliográficos ou bancos de dados que registam o conteúdo da revista em questão), acoplamento bibliográfico (co-citação e coocorrência) e análise de citações de artigos e patentes científicas. Os métodos bibliométricos são quantitativos por natureza, mas são usados para fazer pronunciamentos sobre características qualitativas. O principal objetivo de todos os tipos de exercícios bibliométricos é transformar algo intangível em uma entidade gerenciável (Wallin, 2005).

Para a obtenção dos dados e informações necessárias ao estudo, foi utilizada a base de dados Scopus, que apresenta maior base (Falagas et al., 2008), maior número de periódicos da área de turismo e hospitalidade (Hall, 2011) e alta correlação de papers e citações (Archambault, 2009) em relação à tradicional base Thomson ISI Web of Knowledge. A busca foi realizada em 27/06/2016, com a utilização das palavras-chave "tourist destination", "destination choice", "tourist decision" e "tourist choice", obtendo-se o número de 13.239 documentos. Esse resultado foi restringido através da escolha da área "business, management and Accouting" (resultando em 6.753 documentos) e do tipo de documento, selecionando-se somente os artigos científicos, resultando em 5.760 artigos. Na sequência, foram selecionados somente artigos publicados em Journals classificados no primeiro quartil (Q1) do SClmago Journal Rank indicator (SCImago, 2015) na categoria Tourism, Leisure and Hospitality Management: Tourism Management, Annals of Tourism Research, Journal of Sustainable Tourism, Asia Pacific Journal of Tourism Research, Journal of Travel And Tourism Marketing, International Journal of Tourism

Tab. 1

Scimago Journal Ranking

\begin{tabular}{|c|c|c|c|c|c|c|c|}
\hline \# & Titulo & SJR & $\begin{array}{c}\text { SJR } \\
\text { Quartile }\end{array}$ & $\begin{array}{c}\mathrm{H} \\
\text { index }\end{array}$ & $\begin{array}{c}\text { Artigos } \\
\text { Publicados } \\
2015\end{array}$ & $\begin{array}{c}\text { Artigos } \\
\text { Publicados } \\
2012-2014\end{array}$ & $\begin{array}{l}\text { Citações } \\
\text { recebidas } \\
2012-2014\end{array}$ \\
\hline 1 & Annals of Tourism Research & 2,658 & Q1 & 108 & 80 & 330 & 1090 \\
\hline 2 & Tourism Management & 2,45 & Q1 & 110 & 195 & 493 & 2127 \\
\hline 3 & Journal of Sustainable Tourism & 1,995 & Q1 & 60 & 95 & 196 & 634 \\
\hline 4 & International Journal of Hospitality Management & 1,887 & Q1 & 60 & 138 & 482 & 1449 \\
\hline 5 & Cornell Hospitality Quarterly & 1,862 & Q1 & 47 & 39 & 125 & 254 \\
\hline 6 & Journal of Hospitality and Tourism Research & 1,729 & Q1 & 40 & 23 & 74 & 188 \\
\hline 7 & Journal of Service Management & 1,506 & Q1 & 27 & 35 & 94 & 365 \\
\hline 8 & Cities & 1,422 & Q1 & 49 & 148 & 363 & 1010 \\
\hline 9 & International Journal of Contemporary Hospitality Management & 1,329 & Q1 & 35 & 83 & 172 & 459 \\
\hline 10 & Applied Geography & 1,306 & Q1 & 52 & 222 & 610 & 2007 \\
\hline 11 & Journal of Travel and Tourism Marketing & 1,28 & Q1 & 26 & 122 & 169 & 439 \\
\hline 12 & Journal of Hospitality and Tourism Management & 1,12 & Q1 & 14 & 14 & 40 & 54 \\
\hline 13 & Journal of Vacation Marketing & 1,091 & Q1 & 40 & 26 & 83 & 157 \\
\hline 14 & Tourism Management Perspectives & 1,067 & Q1 & 13 & 71 & 141 & 328 \\
\hline 15 & International Journal of Tourism Research & 1,064 & Q1 & 24 & 76 & 166 & 270 \\
\hline 16 & Journal of Hospitality Marketing and Management & 1,032 & Q1 & 20 & 67 & 128 & 244 \\
\hline 17 & Asia Pacific Journal of Tourism Research & 0,921 & Q1 & 18 & 116 & 168 & 259 \\
\hline 18 & International Journal of Sport Policy & 0,895 & Q1 & 11 & 46 & 89 & 139 \\
\hline 19 & Sport Management Review & 0,805 & Q1 & 28 & 53 & 135 & 249 \\
\hline 20 & Journal of Place Management and Development & 0,802 & Q1 & 6 & 16 & 37 & 79 \\
\hline
\end{tabular}

Fonte: Scimago (2015) 


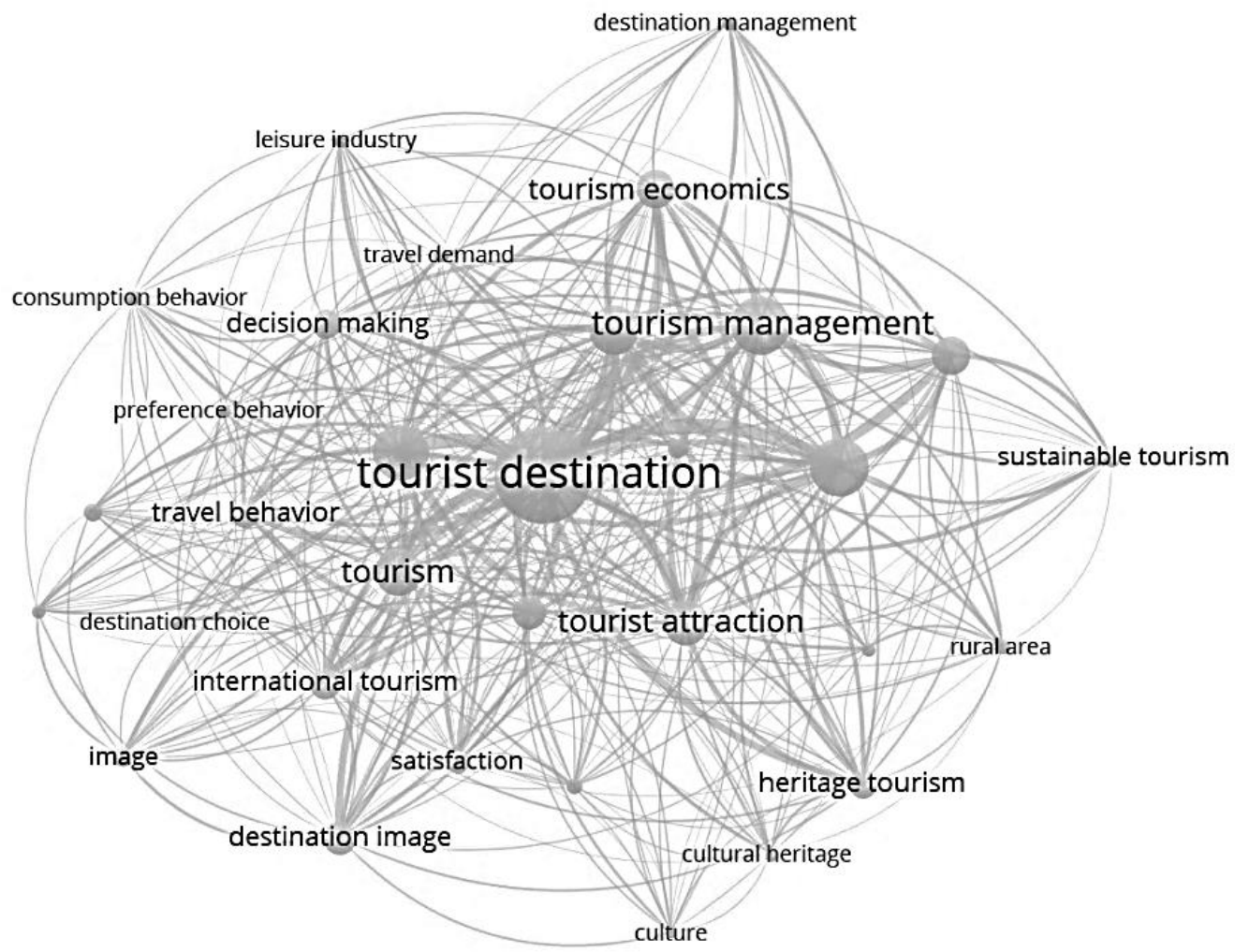

Fig. 1

Mapa dos principais agrupamentos da amostra de 2000 artigos.

Fonte: Processado no VOSViewer (Van Eck e Waltman, 2010) com dados da base Scopus.

Research, Journal of Vacation Marketing, Tourism Management Perspectives ou International Journal of Hospitality Management, Journal of Hospitality Marketing And Management, Journal of Hospitality And Tourism Research, International Journal of Contemporary Hospitality Management. A restrição baseada nos principais Journals da área segundo o SCImago Journal Ranking - SJR (Tabela 1) resultou em 2.222 artigos, que foram ordenados pela quantidade de citações indicadas na base de dados e selecionados os primeiros 2.000 artigos mais citados (limite máximo estabelecido pela base de dados Scopus para a exportação de dados em um único arquivo) para a composição da amostra para a primeira análise. A utilização da citação como critério de seleção dos artigos da amostra é baseada nos argumentos defendidos por Bornmann et al. (2008), para quem citações são indicadores de impacto da pesquisa, ou seja, o grau em que ela tem sido útil para outros pesquisadores, e por Van Raan (2004) para quem análise bibliométrica baseada em quantidade de citações fornece indicadores de impacto e influência internacional.

A primeira analise da amostra foi realizada com a utilização do software estatístico SciMat (Cobo et al., 2012). Informações relevantes podem ser obtidas a partir da frequência de co-ocorrência de palavraschave, extraídas da base de dados por meio da contagem do número de documentos em que as duas palavras-chave aparecem juntos. No final do processo, são obtidos conjuntos de agrupamentos (clusters) que podem ser entendidos como grupos semânticos ou conceituais dos diferentes temas tratados pelo campo de pesquisa, podendo ser utilizados com várias finalidades, entre elas a quantificação do campo de pesquisa (Cobo et al., 2011). De outra forma, pode-se dizer que o software identifica as palavras-chave de cada artigo e forma agrupamentos com base nessas palavras-chave, onde as distâncias entre os agrupamentos são definidas pela frequência em que as palavras-chave ocorrem em conjunto nos registros do banco de dados. Por exemplo, agrupamentos formados pelas palavraschave "decision making" e "travel behaviour" podem estar mais próximos ou distantes, dependendo do número de vezes em que ambas palavras-chave são encontradas nos artigos que compõem esses agrupamentos.

$\mathrm{Na}$ amostra, foram encontrados 6.282 agrupamentos baseados em co-ocorrência de palavras-chave. Os principais clusters e as distâncias entre eles podem ser visualizados na figura 1. 
Na busca de clusters mais relevantes para o estudo, 6.065 agrupamentos foram excluídos, pois foram baseadas em palavras-chave não significativas para o estudo (por exemplo, nome de países, áreas da administração e denominações de métodos de pesquisa), além de serem grupos compostos por menos de 10 artigos. Dos 217 clusters restantes, 2 clusters apresentaram maior aderência e concentração de artigos relevantes sobre o tema da pesquisa, sendo portanto escolhidos para a análise com maior profundidade: "decison making" (com 112 artigos) e "destination choice" (com 36 artigos). Por fim, esses artigos foram identificados, separados e, após a eliminação dos registros duplicados, formaram a amostra final de 141 artigos utilizada como fonte das análises e resultados apresentados nos próximos tópicos.

\section{RESULTADOS DA PESQUISA}

Com base na amostra de 141 artigos, foram realizados análises e levantamento de indicadores bibliométricos para maior entendimento do tema abordado no estudo. Em relação ao ano das publicações, verifica-se uma tendência de crescimento no número de artigos publicados nos journals selecionados a partir de 1997, atingindo o auge no ano de 2012 e decrescendo novamente até 2015 (Figura 2). Até 1996, poucos artigos foram publicados anualmente e a maioria dos artigos mais citados na base de dados foram publicados entre os anos 2000 e 2010 (Tabela 2). Os dados indicam que esse é um tema de interesse recente, com publicações de maior impacto concentradas na última década.

Tab. 2

Num. de publicações em journals com maior relevância por ano.

\begin{tabular}{lll}
\hline Ano & N & $\%$ \\
\hline 1986 & 1 & 0,7 \\
1987 & 0 & 0,0 \\
1988 & 0 & 0,0 \\
1989 & 0 & 0,0 \\
1990 & 1 & 0,7 \\
1991 & 0 & 0,0 \\
1992 & 1 & 0,7 \\
1993 & 1 & 0,7 \\
1994 & 0 & 0,0 \\
1995 & 1 & 0,7 \\
1996 & 1 & 0,7 \\
1997 & 2 & 1,4 \\
1998 & 4 & 2,8 \\
1999 & 1 & 0,7 \\
2000 & 4 & 2,8 \\
2001 & 1 & 0,7 \\
\hline
\end{tabular}

\begin{tabular}{ccc}
\hline 2002 & 1 & 0,7 \\
2003 & 3 & 2,1 \\
2004 & 6 & 4,3 \\
2005 & 4 & 2,8 \\
2006 & 5 & 3,5 \\
2007 & 6 & 4,3 \\
2008 & 8 & 5,7 \\
2009 & 11 & 7,8 \\
2010 & 12 & 8,5 \\
2011 & 13 & 9,2 \\
2012 & 20 & 14,2 \\
2013 & 13 & 9,2 \\
2014 & 9 & 6,4 \\
2015 & 8 & 5,7 \\
2016 & 4 & 2,8 \\
\hline Total & 141 & 100 \\
\hline
\end{tabular}

Fonte: Elaborado pelo autor com dados da base Scopus.

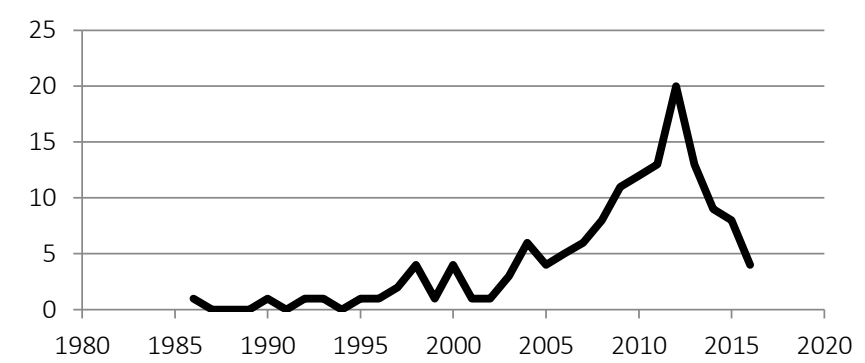

Fig. 2

Crescimento do número de publicações ao longo do tempo. Fonte: Elaborado pelo autor com dados da base Scopus.

Em relação à fonte da publicação, cinco journals concentraram 90,1\% das publicações, com destaque para o Tourism Management Journal com 41,8\% das publicações (Tabela 3).

Tab.3

Número de artigos publicados por journal

\begin{tabular}{lcc}
\hline \multicolumn{1}{c}{ Fonte } & N & $\%$ \\
\hline Tourism Management & 59 & 41,8 \\
Annals of Tourism Research & 32 & 22,7 \\
Asia Pacific Journal of Tourism Research & 15 & 10,6 \\
International Journal of Tourism Research & 13 & 9,2 \\
Journal of Sustainable Tourism & 8 & 5,7 \\
Journal of Travel and Tourism Marketing & 4 & 2,8 \\
Journal of Vacation Marketing & 4 & 2,8 \\
Int. Journal of Contemporary Hospitality Management & 2 & 1,4 \\
Environment Development and Sustainability & 1 & 0,7 \\
Journal of Hospitality and Tourism Research & 1 & 0,7 \\
Journal of Hospitality Marketing and Management & 1 & 0,7 \\
Tourism Management Perspectives & 1 & 0,7 \\
\hline Total & 141 & 100 \\
\hline
\end{tabular}

Fonte: Elaborado pelo autor com utilização de dados da Scopus.

Não existe uma grande concentração quando considerado o ranking das instituições a qual estão ligadas as pesquisas. As instituições com maior número de publicações possuem no máximo 4 a 3 trabalhos publicados, com exceção da School of Hotel 
and Tourism Management, com 7 artigos publicados (Tabela 4). Uma avaliação realizada na base de dados aponta que a maioria das instituições listadas estão relacionadas a um único artigo.

Tab. 4

Artigos publicados por instituição de ensino.

\begin{tabular}{lcc}
\hline Instituições & N & \% \\
\hline School of Hotel and Tourism Management Hong Kong & 7 & 3,0 \\
University of Queensland & 5 & 2,1 \\
Hong Kong Polytechnic University & 4 & 1,7 \\
Purdue University & 4 & 1,7 \\
University of Florida & 4 & 1,7 \\
Mugia Universitesi & 4 & 1,7 \\
Curtin University & 4 & 1,7 \\
Universidad de Malaga & 3 & 1,3 \\
Lincoln University, New Zealand & 3 & 1,3 \\
Universidad de Las Palmas de Gran Canaria & 3 & 1,3 \\
Universitat d'Alacant & 3 & 1,3 \\
University of Wollongong & 3 & 1,3 \\
Monash University & 3 & 1,3 \\
Copenhagen Business School & 3 & 1,3 \\
Texas A and M University & 3 & 1,3 \\
Bournemouth University & 3 & 1,3 \\
Temple University & 3 & 1,3 \\
Rosen College of Hospitality Management & 3 & 1,3 \\
Outras instituições & 168 & 72,1 \\
\hline Total & 233 & 100 \\
\hline
\end{tabular}

Fonte: Elaborado pelo autor com utilização de dados da Scopus
Uma análise de conteúdo, de cunho qualitativo, foi então realizada no resumo dos 24 artigos mais citados da amostra (Tabela 5), ou seja, artigos com até $1 \%$ do total das citações. Como resultado, temos os principais assuntos tratados nesse grupo:

- Modelos de decisão ou escolha de destino (5 artigos);

- Fatores motivadores para turismo (4 artigos);

- Características ou fatores pessoais determinantes na escolha do destino (4 artigos);

- Influência da informação e comunicação na escolha do destino (4 artigos);

- Influência da imagem do destino (2 artigos).

Ainda com base na amostra, foi realizada uma análise de agrupamentos com a utilização do software estatístico SciMat (Cobo et al., 2012). Foram encontrados 805 agrupamentos baseados em coocorrência de palavras-chave. Os principais clusters podem ser visualizados na Figura 3 e a quantidade de artigos em cada cluster apresentados na Tabela 6.

Como o tema do estudo é a escolha de destino, foi realizada uma nova análise de conteúdo, de cunho qualitativo, junto aos resumos dos 35 artigos do

Tab. 5

Top 24 artigos mais citados da amostra

\begin{tabular}{|c|c|c|c|}
\hline Autores & Título do Artigo & $\mathrm{N}$ & $\%$ \\
\hline Um S., Crompton J.L. & Attitude determinants in tourism destination choice & 387 & 8,5 \\
\hline Sönmez S.F., Graefe A.R & Influence of terrorism risk on foreign tourism decisions & 240 & 5,3 \\
\hline Vogt C.A., Fesenmaier D.R & Expanding the functional information search model & 214 & 4,7 \\
\hline Fodness D., Murray B. & Tourist information search & 211 & 4,7 \\
\hline Sirakaya E., Woodside A.G. & Building and testing theories of decision making by travellers & 211 & 4,7 \\
\hline Goossens C. & Tourism information and pleasure motivation & 183 & 4,0 \\
\hline Phelps A. & Holiday destination image - the problem of assessment. & 151 & 3,3 \\
\hline Benítez J.M. et al. & Using fuzzy number for measuring quality of service in the hotel industry & 128 & 2,8 \\
\hline Hollinshead K. & Surveillance of the worlds of tourism: Foucault and the eye-of-power & 116 & 2,6 \\
\hline Oppermann M. & Travel life cycle & 99 & 2,2 \\
\hline Bansal H., Eiselt H.A. & Exploratory research of tourist motivations and planning & 97 & 2,1 \\
\hline Divisekera S & A model of demand for international tourism & 88 & 1,9 \\
\hline Lepp A., Gibson H. & Sensation seeking and tourism: Tourist role, perception of risk and destination choice & 86 & 1,9 \\
\hline Molina A., Esteban A. & Tourism Brochures. Usefulness and Image & 84 & 1,9 \\
\hline Gokovali U. et al. & Determinants of length of stay: A practical use of survival analysis & 83 & 1,8 \\
\hline Decrop A., Snelders D. & A grounded typology of vacation decision-making & 83 & 1,8 \\
\hline Moscardo G. et Al. & Understanding vacation destination choice through travel motivation and activities & 78 & 1,7 \\
\hline Decrop A., Snelders D. & Planning the summer vacation - An adaptable process & 70 & 1,5 \\
\hline d'Angella F., Go F.M. & Tale of two cities' collaborative tourism marketing & 60 & 1,3 \\
\hline Yüksel A., Akgül O. & Postcards as affective image makers: An idle agent in destination marketing & 57 & 1,3 \\
\hline Nicolau J.L., Más F.J. & Stochastic modeling. A three-stage tourist choice process & 56 & 1,2 \\
\hline Slade P. & Gallipoli thanatourism: The meaning of ANZAC & 56 & 1,2 \\
\hline Moscardo G. & Shopping as a destination attraction & 56 & 1,2 \\
\hline Jacobsen J.K.S., Munar A.M. & Tourist information search and destination choice in a digital age & 45 & 1,0 \\
\hline Outros autores & Títulos & 1595 & $<1$ \\
\hline Total de Citações & & 4534 & 100 \\
\hline
\end{tabular}

Fonte: Elaborado pelo autor com utilização de dados da base Scopus. 


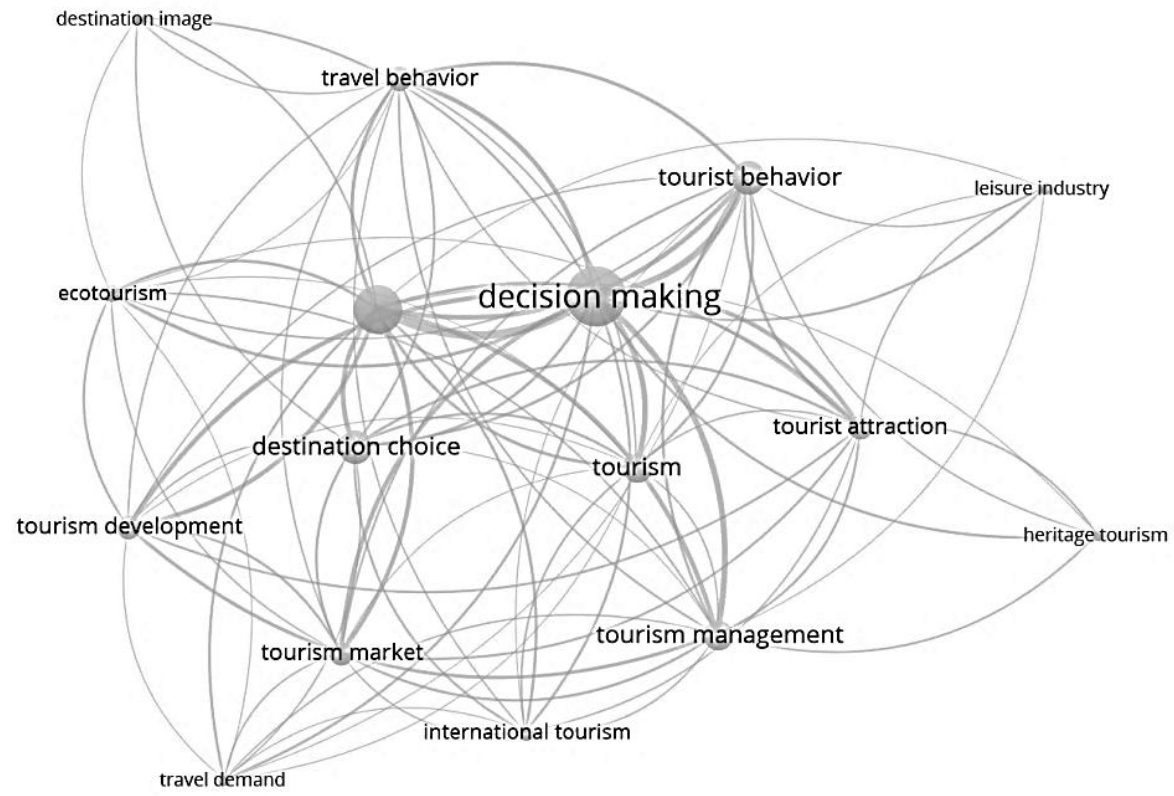

Fig. 3

Mapa dos principais agrupamentos e relações da amostra de artigos selecionados.

Fonte: Processado no VOSViewer (Van Eck \& Waltman, 2010) com dados da base Scopus.

cluster "destination choice" (Tabela 7). Os principais assuntos tratados pelos artigos nesse cluster são:

- Modelos de decisão ou escolha de destino (5 artigos);

- Fatores motivadores para turismo (6 artigos);

- Características ou fatores pessoais determinantes na escolha do destino (7 artigos);

- Características e atrativos do destino determinantes na escolha do destino (3 artigos);

- Influência da imagem do destino (3 artigos);

- Influência da informação e comunicação na escolha do destino (2 artigos).

Tab. 6

Número de artigos por Cluster

\begin{tabular}{lc} 
& Clusters \\
\hline Decision-Making & N \\
Tourist-Destination & 69 \\
Tourist-Behavior & 36 \\
Destination-Choice & 35 \\
Tourism & 28 \\
Tourism-Management & 28 \\
Tourism-Development & 19 \\
Travel-Behavior & 19 \\
Tourism-Market & 18 \\
Tourist-Attraction & 18 \\
Ecotourism & 9 \\
International-Tourism & 8 \\
Heritage Tourism & 5 \\
Leisure Industry & 5 \\
\hline
\end{tabular}

Fonte: Compilação realizada no SciMat (Cobo et al., 2012) com dados da base Scopus.
As análises de conteúdo realizadas tanto nos artigos de maior relevância da amostra inicial (2.000 artigos) quanto na totalidade do cluster "destination choice" (35 artigos) apresentam resultados similares em relação aos assuntos tratados. Portanto, considerando as duas análises, há indícios de que os principais assuntos abordados em pesquisas relacionadas ao tema "escolha de destino turístico" são: (a) modelos de decisão ou escolha de destino, (b) fatores motivadores para turismo, (c) características ou fatores pessoais determinantes na escolha do destino, (d) características e atrativos do destino determinantes na escolha do destino, (e) influência da imagem do destino e (f) influência da informação e comunicação na escolha do destino. Esses assuntos, em partes ou na totalidade, podem ser utilizados por pesquisadores que queiram realizar um estudo completo sobre o tema. Os itens (a), (b), (c) e (d) são mencionados no estudo realizado por Sirakaya e Woodside (2005) como principais componentes dos modelos de tomada de decisão em turismo, enquanto os itens (e) e (f) não foram destacados no mesmo estudo. Em relação aos itens (e) influência da imagem do destino e (f) influência da informação e comunicação na escolha do destino, é importante ressaltar que apesar de alguns artigos (Phelps,1986; Fodness \& Murray, 1997; Vogt \& Fesenmaier, 1998; Decrop \& Snelders, 2004) serem publicados antes do estudo, a maioria dos artigos que tratam desses assuntos (Prentice \& Andersen, 2000; Glover, 2011; Kerr, Cliff \& Dolnicar, 2011; Park \& Nicolau, 2015; 
Tab. 7

Artigos do cluster "Destination Choice"

\begin{tabular}{|c|c|c|c|}
\hline Autores & Artigo & $\mathrm{N}$ & $\%$ \\
\hline Um S., Crompton J.L. & Attitude determinants in tourism destination choice & 387 & 23,7 \\
\hline Sönmez S.F., Graefe A.R. & Influence of terrorism risk on foreign tourism decisions & 240 & 14,7 \\
\hline Oppermann M. & Travel life cycle & 99 & 6,1 \\
\hline Divisekera S. & A model of demand for international tourism & 88 & 5,4 \\
\hline Lepp A., Gibson H. & Sensation seeking and tourism: Tourist role, perception of risk and destination choice & 86 & 5,3 \\
\hline Molina A., Esteban A. & Tourism Brochures. Usefulness and Image & 84 & 5,1 \\
\hline Moscardo G. et al. & Understanding vacation destination choice through travel motivation and activities & 78 & 4,8 \\
\hline Decrop A., Snelders D. & Planning the summer vacation - An adaptable process & 70 & 4,3 \\
\hline Yüksel A., Akgül O. & Postcards as affective image makers: An idle agent in destination marketings & 57 & 3,5 \\
\hline Moscardo G. & Shopping as a destination attraction & 55 & 3,4 \\
\hline Jacobsen, J.K.S., Munar, A.M. & Tourist information search and destination choice in a digital age & 45 & 2,8 \\
\hline Eugenio-Martin J.L., Campos-Soria J.A. & Climate in the region of origin and destination choice in outbound tourism demand & 41 & 2,5 \\
\hline Prentice R., Andersen V. & Evoking Ireland: Modeling tourist propensity & 41 & 2,5 \\
\hline Correia A., Santos C.M., Barros C.P. & Tourism in Latin America A Choice Analysis & 32 & 2,0 \\
\hline Sirakaya E., Sheppard A.G., McLellan R.W. & Assessment of the relationship between perceived safety at a vacation site and destination choice decisions & 27 & 1,7 \\
\hline Reichel A., Fuchs G., Uriely N. & Israeli Backpackers. The Role of Destination Choice & 23 & 1,4 \\
\hline Reisinger Y., Turner L. & Japanese tourism satisfaction: Gold Coast versus Hawaii & 22 & 1,3 \\
\hline Llewellyn-Smith C., McCabe V.S. & What is the attraction for exchange students: The host destination or host university? & 20 & 1,2 \\
\hline Wong J.-Y. et al. & Identifying valuable travelers and their next foreign destination by the application of data mining techniques & 20 & 1,2 \\
\hline Seaton A.V. & Social stratification in tourism choice and experience since the war & 16 & 1,0 \\
\hline Martin B., Mason S & The future for attractions. Meeting the needs of the new consumers & 15 & 0,9 \\
\hline Moghimehfar F., Nasr-Esfahani M.H. & Decisive factors in medical tourism destination choice: A case study of Isfahan, Iran and fertility treatments & 15 & 0,9 \\
\hline Taplin J.H.E., McGinley C. & A linear program to model daily car touring choices & 14 & 0,9 \\
\hline Eugenio-Martin J.L., Campos-Soria J.A. & Economic crisis and tourism expenditure cutback decision & 12 & 0,7 \\
\hline Guillet B.D., Lee A., Law R., Leung R. & Factors affecting outbound tourists' destination choice: The case of Hong Kong & 9 & 0,6 \\
\hline Nicolau J.L. & Differentiated price loss aversion in destination choice: The effect of tourists' cultural interest & 9 & 0,6 \\
\hline Glover P. & International students: Linking education and travel & 5 & 0,3 \\
\hline Huang J., Cai L.A. & Destination choice model for transitional travel: College students in China & 4 & 0,2 \\
\hline Kerr G., Cliff K., Dolnicar S. & Harvesting the "'Business Test Trip"': Converting Business Travelers to Holidaymakers & 4 & 0,2 \\
\hline Lee G., Tussyadiah I.P. & Exploring Familiarity and Destination Choice in International Tourism & 3 & 0,2 \\
\hline Oppewal H., Huybers T., Crouch G.I. & Tourist destination and experience choice: A choice experimental analysis of decision sequence effects & 3 & 0,2 \\
\hline Yang Y., Fik T., Zhang J. & Modeling sequential tourist flows: Where is the next destination? & 3 & 0,2 \\
\hline Choi H.C., Lee S. & Understanding US traveler behavior to Asian countries: A secondary analysis approach & 2 & 0,1 \\
\hline Sharifpour M., Walters G., Ritchie B.W. & Risk perception, prior knowledge, and willingness to travel & 2 & 0,1 \\
\hline Park S., Nicolau J.L. & Differentiated effect of advertising: Joint vs. separate consumption & 1 & 0,1 \\
\hline Total & & 1632 & 100 \\
\hline
\end{tabular}

Fonte: Compilação realizada no SciMat (Cobo et al., 2012) com dados da base Scopus.

Molina \& Esteban, 2006; Yüksel \& Akgül, 2007; Jacobsen \& Munar, 2012) foram publicados em data posterior ao levantamento realizado por Sirakaya e Woodside (2005). Da mesma forma, os grupos de fatores determinantes para a escolha de destino identificados por esses autores como "natureza da viagem pretendida" (tamanho do feriado, distância, duração da viagem, etc.) e "experiências da viagem" (humor e sentimentos durante a viagem, avaliações pós-viagem, etc.), não foram destacados tanto na formação de cluster quanto na análise de conteúdo realizada, apesar de haver artigos relativos a esses assuntos na amostra coletada.

\subsection{Análise de Citações provenientes da amostra}

Nesse tópico são apresentadas as análises de citações provenientes da amostra, ou seja, análises realizadas com base na citações de artigos e autores dentro dos artigos que integram a amostra.
Para Bornmann et al. (2008) a publicação de um trabalho de pesquisa serve para disseminar os resultados da pesquisa, convidando outros cientistas a usar os resultados em suas próprias pesquisas e indicando isso por meio de uma citação formal. Como as citações são destinadas a mostrar que uma publicação utilizou o conteúdo de outras publicações (resultados e ideias), as contagens de citações são utilizadas como indicador de impacto da pesquisa, ou seja, o grau em que ela tem sido útil para outros pesquisadores. Da mesma forma, Van Raan (2004) afirma que análise bibliométrica baseada em citações fornece indicadores de impacto e influência internacional, pois contagens de citações estão sendo utilizadas para avaliação e comparação da pesquisa desempenho dos investigadores individuais, departamentos e instituições de pesquisa, bem como o impacto científico das nações.

A análise de citações, porém, apresenta limitações. Bornmann e Daniel (2008) afirmam que 
Tab. 8

Artigos mais citados pelos artigos provenientes do cluster "Destination Choice"

\begin{tabular}{|c|c|c|c|c|}
\hline Autores & Artigo & Ano & $\mathrm{N}$ & $\%$ \\
\hline Woodside, A.G., Lysonski, S. & A general model of traveller destination choice & 1989 & 28 & 0,35 \\
\hline Um, S., Crompton, J.L.. & Attitude determinants in tourism destination choice & 1990 & 25 & 0,32 \\
\hline Moutinho, L.. & Consumer behaviour in tourism & 1987 & 24 & 0,30 \\
\hline Crompton, J.L. & Motivations for Pleasure Vacation & 1979 & 23 & 0,29 \\
\hline $\begin{array}{r}\text { Engel, J.F., Kollat, D.T., } \\
\text { Blackwell, R.D. }\end{array}$ & Consumer Behavior & 1978 & 21 & 0,27 \\
\hline Hair, J.F. et. Al. & Multivariate data analysis & 1998 & 20 & 0,25 \\
\hline Sirakaya, E., Woodside, A.G. & Building and testing theories of decision-making by travellers & 2005 & 19 & 0,24 \\
\hline Mansfild, Y. & From Motivation to Actual Travel & 1992 & 13 & 0,16 \\
\hline Mathieson, A., Wall, G. & Tourism: Economic, physical and social impacts & 1982 & 13 & 0,16 \\
\hline Echtner, M., Ritchie, B., & The measurement of destination image: An empirical assessment & 1993 & 12 & 0,15 \\
\hline Fodness, D., Murray, B. & Tourist information search & 1997 & 12 & 0,15 \\
\hline Crompton, J.L., Ankomah, P.K. & Choice set propositions in destination decisions & 1993 & 11 & 0,14 \\
\hline Decrop, A., & Tourists' decision-making behavior processes & 1999 & 11 & 0,14 \\
\hline Fesenmaier, D.R., Jeng, J.-M., & Assessing structure in the pleasure trip planning process & 2000 & 11 & 0,14 \\
\hline Gursoy, D., McCleary, K.W., & An integrative model of tourist's information search behaviour & 2004 & 11 & 0,14 \\
\hline Howard, J.A., Sheth, J.N., & The Theory of Buyer Behaviour & 1969 & 11 & 0,14 \\
\hline Plog, S.C., & Why destination areas rise and fall in popularity & 1974 & 11 & 0,14 \\
\hline Decrop, A., Snelders, D. & Planning the summer vacation: An adaptable process & 2004 & 10 & 0,13 \\
\hline Fodness, D. & The impact of the family life-cycle on the vacation decision-making process & 1992 & 10 & 0,13 \\
\hline Gartner, W.C., & Image formation process & 1993 & 10 & 0,13 \\
\hline Hofstede, G., & Culture's Consequences: International Differences in Work-Related Values & 1980 & 10 & 0,13 \\
\hline Decrop, A., Snelders, D., & A grounded typology of vacation decision-making & 2005 & 9 & 0,11 \\
\hline Mayo, E., Jarvis, L.. & The Psychology of Leisure Travel: Effective Marketing and Selling of Travel Services & 1981 & 9 & 0,11 \\
\hline Milman, A., Pizam, A. & The Role of Awareness and Familiarity with Destination: The Central Florida Case & 1995 & 9 & 0,11 \\
\hline Um, S. & The roles of perceived inhibitors and perceived facilitators in the pleasure travel destination choice process & 1990 & 9 & 0,11 \\
\hline Anderson, J.C., Gerbing, D.W. & Structural equation modeling in practice: A review and recommended two-step approach & 1988 & 8 & 0,10 \\
\hline Cohen, E. & Towards a sociology of international tourism & 1972 & 8 & 0,10 \\
\hline Fishbein, M., Ajzen, I. & Beliefs, Attitude, Intention and Behavior: An Introduction to Theory and Research & 1975 & 8 & 0,10 \\
\hline Fodness, D., Murray, B., & A model of tourist information search behavior & 1999 & 8 & 0,10 \\
\hline Fornell, C., Larcker, D.F., & Structural equation models with unobservable variables and measurement error: Algebra and statistics & 1981 & 8 & 0,10 \\
\hline Ajzen, I., Fishbein, M., & Understanding Attitudes and Predicting Social Behaviour & 1980 & 7 & 0,09 \\
\hline Total & & & 7907 & 100,00 \\
\hline
\end{tabular}

Fonte: Cruzamento de dados obtidos na Base Scopus

citações não são motivadas apenas pelo desejo de reconhecer influências intelectuais e cognitivas de colegas cientistas, uma vez que os estudos individuais revelam também outros fatores não-científicos que desempenham um papel na decisão de citar. Por exemplo, fatores sociais, localização e prestígio do autor, idioma e disponibilidade de revistas para publicação (Bornmann et al., 2008). Apesar disso, medidas de citação tem demonstrado ser uma forma válida de julgamento de pares que introduz um elemento de objetividade útil para o processo de avaliação e envolve apenas uma pequena fracção do custo de levantamento técnicas (Garfield, 1979).

$\mathrm{Na}$ Tabela 8 estão apresentados os artigos mais citados pelos autores nos artigos do cluster "destination choice". Além dos principais assuntos já identificados e discutidos nesse estudo, destaca-se a inserção de outros assuntos como análises estatísticas (Hair et al., 1998; Fornell \& Larcker, 1981), comportamento do consumidor (Engel et al., 1973; Howard \& Sheth, 1969) além de fatores psicológicos (Mayo \& Jarvis, 1981; Fishbein \& Ajzen,
1975; Ajzen \& Fishbein, 1980) e fatores culturais (Hofstede, 1980) que afetam o comportamento do consumidor ou, especificamente, do turista.

Entre os autores mais citados nos artigos que compõem a amostra, destacam-se Crompton e Woodside, autores dos principais artigos citados (tabela 9). Percebe-se também a presença de autores (e artigos) da área de turismo que são muito citados, mas não constam no cluster pesquisado, entre eles: Daniel R. Fesenmaier, J.R. Brent Ritchie, Dale Fodness, Abraham Pizam e Luiz Moutinho.

\subsection{Análise de Co-citações}

Nesse tópico será apresentado o resultado da análise de co-citações baseados nas referências indicadas nos artigos do cluster "destination choice". Para Small (1973), co-citação é a frequência com a qual dois itens anteriores são citados juntos em uma literatura posterior. O número de citações de dois itens idênticos define a força da co-citação e consequentemente o grau de relação ou associação 
entre dois artigos, ou seja, como são percebidos por uma população de autores. Portanto, os padrões de co-citação podem usados para mapear em grande detalhe as relações entre conceitos chave, métodos ou experimentos em um campo do conhecimento. Essa visão é reforçada por Wallin (2005) para quem quanto o maior o número de pesquisadores citando as mesmas duas publicações, maior a probabilidade de que a dupla citação não seja um acontecimento fortuito, expressando um tipo de assunto relacionado entre as publicações citadas, estabelecendo relacionamentos visíveis dentro das áreas de investigação e entre disciplinas científicas.

Tab. 9

Autores mais citados nos artigos provenientes do cluster "Destination Choice"

\begin{tabular}{|c|c|}
\hline Autor & Citações \\
\hline Crompton & 175 \\
\hline Woodside & 118 \\
\hline Fesenmaier D.R. & 90 \\
\hline Ritchie J.R.B. & 57 \\
\hline Decrop A. & 50 \\
\hline Ajzen, I. & 45 \\
\hline Sirakaya & 45 \\
\hline Um S. & 41 \\
\hline Fodness D. & 40 \\
\hline Pizam A. & 39 \\
\hline Gartner W.C. & 29 \\
\hline Moutinho & 28 \\
\hline Lisonski & 28 \\
\hline Gursoy D. & 28 \\
\hline Baloglu S. & 26 \\
\hline Engel J.F. & 26 \\
\hline Mansfeld Y. & 26 \\
\hline Wall G. & 25 \\
\hline Plog S. C. & 25 \\
\hline Cohen E. & 25 \\
\hline Fishbein M. & 23 \\
\hline Baker D. & 23 \\
\hline Ankomah & 21 \\
\hline Ankomah P.K. & 21 \\
\hline Blackwell & 21 \\
\hline Howard J. & 21 \\
\hline Anderson R.E. & 20 \\
\hline Hair J. F. & 20 \\
\hline Snelders D. & 19 \\
\hline Black W.C. & 18 \\
\hline Echetner C. & 17 \\
\hline Hofstede G. & 17 \\
\hline Tathan R.L. & 16 \\
\hline Sheth J.N. & 16 \\
\hline Mathieson A. & 14 \\
\hline Milman A. & 14 \\
\hline Fornell C. & 11 \\
\hline Mayo, E. & 11 \\
\hline Kollat D.T. & 10 \\
\hline Gerbing D.W. & 9 \\
\hline Anderson J.C. & 8 \\
\hline
\end{tabular}

Fonte: Cruzamento de dados obtidos na Base Scopus
Na Figura 4 estão apresentadas as principais cocitações baseadas nas referências dos artigos da amostra. A análise cruzada, realizada com os 10 autores mais citados nas referências, demonstrou que John L. Crompton e Arch Woodside são os autores mais citados em conjunto com outros autores. Crompton e Woodside apresentam modelos de processo de decisão e escolha de destinos similares, baseados em formação de conjuntos de opções de destino (choice-sets), cujas escolhas são influenciadas por diversos fatores pessoais e externos.

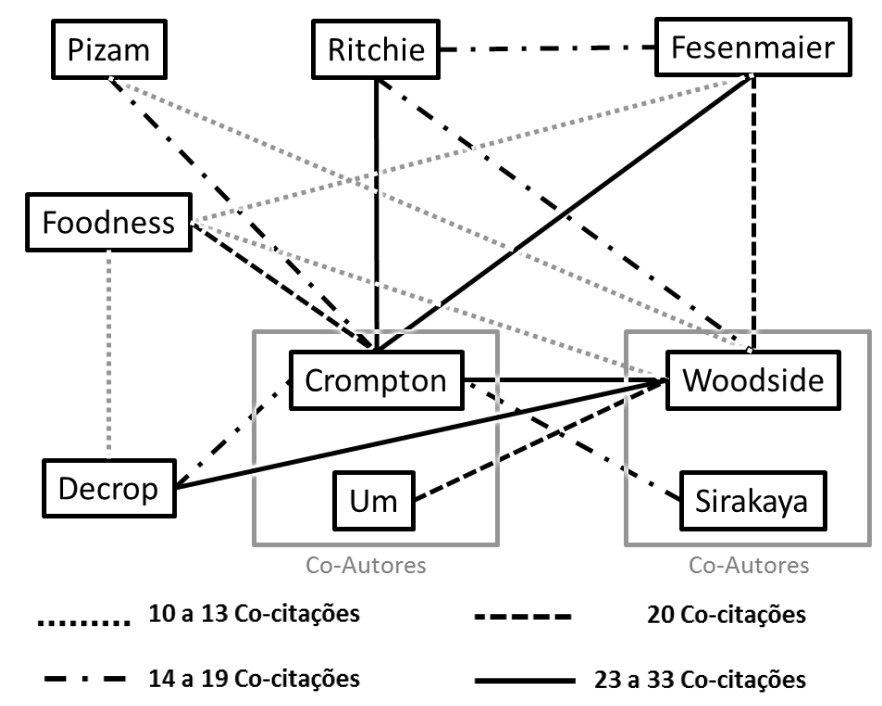

Fig. 4

Mapa de autores co-citados nos artigos da amostra

Fonte: Cruzamento de dados obtidos na Base Scopus.

Em trabalhos envolvendo imagem de destino, com utilização de artigos de Ritchie, autores Crompton, Woodside e Fesenmaier podem ser utilizados para apresentação e discussão de modelos de processo de decisão. Da mesma forma, tanto trabalhos que estudem a lembrança e familiaridade com o destino (citando Pizam) ou que envolvam a influência do processo de informação na escolha do destino (citando Fodness), podem utilizar os trabalhos de Crompton e Woodside como modelo ou discussão do processo de escolha de destino. Finalmente, a relação entre Decrop e os autores Crompton e Woodside pode ser estabelecida devido à posição crítica do primeiro autor em relação demais autores, ou seja, artigos de Decrop podem ser utilizados como fundamentação para a apresentação de críticas aos modelos apresentados por Crompton e Woodside.

\section{CONSIDERAÇÕES FINAIS}

O assunto "escolha de destino" em turismo pode ser estudado sob diferentes perspectivas, utilizando 
abordagens e fundamentações teóricas com origem em diferentes áreas da ciência. Alguns estudos utilizam fundamentos originários da área de Economia, calculando a demanda através de modelos econométricos, mas a maioria das pesquisas na área estudam aspectos psicológicos e sociais envolvidos na tomada de decisão, seja focando o próprio processo de decisão (modelos de comportamento do consumidor) ou estudando as principais variáveis psico-comportamentais e a forma como os turistas realizam julgamentos cognitivos e afetivos antes de chegar à decisão final.

O estudo bibliográfico demonstra a diversidade de perspectivas e abordagens ao identificar agrupamentos de estudos relativos a modelos de decisão ou escolha de destino, fatores motivadores para turismo, características ou fatores pessoais, características e atrativos do destino, natureza da viagem pretendida (tamanho do feriado, distância, duração da viagem, etc.), experiências da viagem (humor, sentimentos durante a viagem, avaliações pós-viagem, etc.), influência da imagem do destino e influência da informação/comunicação na escolha do destino turístico. $O$ estudo também demonstra que apesar de haver autores proeminentes, como Crompton e Woodside, os mesmos não são citados na maioria dos estudos avaliados, fato que comprova a pulverização das referências teóricas nos estudos relacionados ao tema.

Como consequência da diversidade de abordagens e perspectivas sobre o tema, na realização do estudo foi necessária, em diversos momentos, a restrição de artigos para a análise. A primeira restrição foi a escolha de artigos publicados somente nos principais journals segundo o SCImago Journal Ranking (SJR). A segunda restrição, estabelecida pela própria base de dados utilizada, foi a limitação do número de artigos que compõem a amostra, sendo escolhidos para análise os 2.000 primeiros artigos, classificados segundo a quantidade de citações recebidas, do mais citado para o monos citado. Por fim, a última restrição foi a realização da análise de citações e co-citações somente no cluster denominado "destination choice". Com essas restrições, podem haver artigos e autores que discutem assuntos relacionados ao tema que não são contemplados nessa análise, sendo essa a principal limitação da pesquisa. Além disso, existem as limitações da própria técnica de análise de citações apontadas Bornmann e Daniel (2008) segundo os quais citações não são motivadas apenas pelo desejo de reconhecer influências intelectuais e cognitivas de colegas cientistas, uma vez que os estudos individuais revelam também outros fatores não-científicos (por exemplo, fatores sociais, localização e prestígio do autor, idioma e disponibilidade de revistas para publicação) desempenham um papel na decisão de citar. Essas limitações, porém, não invalidam as principais conclusões do estudo; pelo contrário, demonstram que a diversidade de abordagens permite a continuidade e exploração do tema em futuras pesquisas.

\section{REFERÊNCIAS}

- $\quad$ Ajzen, I., \& Fishbein, M. (1980). Understanding attitudes and predicting social behavior. Englewood Cliffs, NJ: Prentice-Hall.

- Archambault, É., Campbell, D., Gingras, Y., \& Larivière, V. (2009). Comparing bibliometric statistics obtained from the Web of Science and Scopus. Journal of the American Society for Information Science and Technology, 60(7), 1320-1326.

- Beerli, A., Meneses, G. D., \& Gil, S. M. (2007). Selfcongruity and destination choice. Annals of Tourism Research, 34(3), 571-587.

- Bekk, M., Spörrle, M., \& Kruse, J. (2016). The benefits of similarity between tourist and destination personality. Journal of Travel Research, 55(8), 1008-1021.

- Boksberger, P., Dolnicar, S., Laesser, C., \& Randle, M. (2011). Self-congruity theory: To what extent does it hold in tourism? Journal of Travel Research, 50(4), 454464.

- Bornmann, L., \& Daniel, H. D. (2008). What do citation counts measure? A review of studies on citing behavior. Journal of Documentation, 64(1), 45-80.

- Bornmann, L., Mutz, R., Neuhaus, C., \& Daniel, H. D. (2008). Citation counts for research evaluation: standards of good practice for analyzing bibliometric data and presenting and interpreting results. Ethics in science and environmental politics, 8(1), 93-102.

- $\quad$ Cobo, M. J., López-Herrera, A. G., Herrera-Viedma, E., \& Herrera, F. (2011). An approach for detecting, quantifying, and visualizing the evolution of a research field: A practical application to the fuzzy sets theory field. Journal of Informetrics, 5(1), 146-166.

- Cobo, M. J., López-Herrera, A. G., Herrera-Viedma, E., \& Herrera, F. (2012). SciMAT: A new science mapping analysis software tool. Journal of the American Society for Information Science and Technology, 63(8), 16091630.

- Correia, Antónia; Pimpão, Adriano. (2008). Decisionmaking processes of Portuguese tourist travelling to South America and Africa. International Journal of Culture, Tourism and Hospitality Research, 2(4), 330373. 
- Cronin, B. (2001). Bibliometrics and beyond: some thoughts on web-based citation analysis. Journal of Information science, 27(1), 1-7.

- Decrop, A. (1999). Tourists' decision-making and behavior processes. In A. Pizam, \& Y. Mansfeld (Eds.), Consumer behavior in travel and tourism (pp. 103-133).

- Decrop, A., \& Snelders, D. (2004). Planning the summer vacation: An adaptable process. Annals of Tourism Research, 31(4), 1008-1030.

- $\quad$ Ekinci, Y., Sirakaya-Turk, E., \& Preciado, S. (2013). Symbolic consumption of tourism destination brands. Journal of Business Research, 66(6), 711-718.

- $\quad$ Engel, J. F., Kollat, D. T., \& Blackwell, R. D. (1973). Consumer behaviour. New York, NY, Holt Reinhardt\& Winston.

- Falagas, M. E., Kouranos, V. D., Arencibia-Jorge, R., \& Karageorgopoulos, D. E. (2008). Comparison of SCImago journal rank indicator with journal impact factor. The FASEB Journal: Official Publication of the Federation of American Societies for Experimental Biology, 22, $2623 e 2628$.

- Fishbein, M., I. Ajzen. (1975). Belief, Attitude, Intention and Behavior: An Introduction to Theory and Research. Addison-Wesley, Reading, MA.

- Fodness, D., \& Murray, B. (1997). Tourist information search. Annals of tourism research, 24(3), 503-523.

- Fornell, C., \& Larcker, D. F. (1981). Structural equation models with unobservable variables and measurement error: Algebra and statistics. Journal of marketing research, 382-388.

- Garfield, E. (1979). Is citation analysis a legitimate evaluation tool? Scientometrics, 1(4), 359-375.

- Glover, P. (2011). International students: Linking education and travel. Journal of Travel \& Tourism Marketing, 28(2), 180-195.

- Hair, J. F., Black, W. C., Babin, B. J., Anderson, R. E., \& Tatham, R. L. (1998). Multivariate data analysis (Vol. 5, No. 3, pp. 207-219). Upper Saddle River, NJ: Prentice hall.

- Hall, C. M. (2011).Publish and perish? Bibliometric analysis, journal ranking and the assessment of research quality in tourism. Tourism Management, 32(1), 16-27.

- Hoftstede, G. (1980). Culture's consequences: International differences in work-related values. Beverly Hills, Cal., and London: Sage.

- Howard, John A. \& Jagdish N. Sheth. (1969). The Theory of Buyer Behavior. New York: Wiley.

Jang, S., \& Cai, L. A. (2002). Travel motivations and destination choice: A study of British outbound market. Journal of Travel \& Tourism Marketing, 13(3), 111-133.

- Jacobsen, J. K. S., \& Munar, A. M. (2012). Tourist information search and destination choice in a digital age. Tourism Management Perspectives, 1, 39-47.
- Karl, M., Reintinger, C., \& Schmude, J. (2015). Reject or select: Mapping destination choice. Annals of Tourism Research, 54, 48-64.

- Kerr, G., Cliff, K., \& Dolnicar, S. (2012). Harvesting the "business test trip": Converting business travelers to holidaymakers. Journal of Travel \& Tourism Marketing, 29(5), 405-415.

- Mayo, E. J., \& Jarvis, L. P. (1981). The psychology of leisure travel. Effective marketing and selling of travel services. CBI Publishing Company, Inc.

- Molina, A., \& Esteban, A. (2006). Tourism brochures: Usefulness and image. Annals of Tourism Research, 33(4), 1036-1056

- Moutinho, L. (1987). Consumer behaviour in tourism. European journal of marketing, 21(10), 5-44.

- Narin, F., \& Hamilton, K. S. (1996). Bibliometric performance measures. Scientometrics, 36(3), 293-310.

- Park, S., \& Nicolau, J. L. (2015). Differentiated effect of advertising: Joint vs. separate consumption. Tourism Management, 47, 107-114.

- Phelps, A. (1986). Holiday destination image-the problem of assessment: An example developed in Menorca. Tourism management, 7(3), 168-180.

- $\quad$ Prentice, R., \& Andersen, V. (2000). Evoking Ireland: modeling tourism propensity. Annals of Tourism Research, 27(2), 490-516.

- Sampaio, S. (2013) Estudar o turismo hoje: para uma revisão crítica dos estudos de turismo. Etnográfica [Online], vol. 17. Retrieved September 01, 2015 from http:// etnografica.revues.org/2615.

- SCImago (2015) SJR. - SCImago Journal \& Country Rank. Retrieved July 21, 2015, from http://www.scimagojr.com

- Sirakaya, Ercan; Woodside, Arch G. (2005) Building and testing theories of decision making by travellers. Tourism management, 26 (6). 815-832

- $\quad$ Sirgy, M. J., Grewal, D., Mangleburg, T. F., Park, J. O., Chon, K. S., Claiborne, C. B. \& Berkman, H. (1997). Assessing the predictive validity of two methods of measuring self-image congruence. Journal of the academy of marketing science, 25(3), 229.

- Small, H. (1973). Co-citation in the scientific literature: A new measure of the relationship between two documents. Journal of the American Society for information Science, 24(4), 265-269.

- Stone, M. J. (2016). Deciding not to choose: Delegation to social surrogates in tourism decisions. Tourism Management, 57, 168-179.

- Shu, M., \& Scott, N. (2014). Influence of social media on Chinese students' choice of an overseas study destination: An information adoption model perspective. Journal of Travel \& Tourism Marketing, 31(2), 286-302. 
- Um, S., \& Crompton, J. L. (1990). Attitude determinants in tourism destination choice. Annals of tourism research, 17(3), 432-448.

- UNWTO. (2015). Tourism highlights. Madrid, Spain: United Nations World Tourism Organization.

- Usakli, A., \& Baloglu, S. (2011). Brand personality of tourist destinations: An application of self-congruity theory. Tourism management, 32(1), 114-127.

- Van Eck, N. J., \& Waltman, L. (2010). Software survey: VOSviewer, a computer program for bibliometric mapping. Scientometrics, 84(2), 523-538.

- Van Raan, A. F. (2004). Measuring science. In Handbook of quantitative science and technology research (pp. 1950). Springer Netherlands.
- Vogt, C. A., \& Fesenmaier, D. R. (1998). Expanding the functional information search model. Annals of Tourism Research, 25(3), 551-578.

- Wallin, J. A. (2005). Bibliometric methods: pitfalls and possibilities. Basic \& clinical pharmacology \& toxicology, 97(5), 261-275.

- Woodside, A. G., \& Lysonski, S. (1989). A general model of traveler destination choice. Journal of travel Research, 27(4), 8-14

- Yüksel, A., \& Akgül, O. (2007). Postcards as affective image makers: An idle agent in destination marketing. Tourism Management, 28(3), 714-725.

\section{SOBRE OS AUTORES}

- Claudio Sunao Saito é Graduado e Mestre em Administração pela Pontifícia Universidade Católica de São Paulo, PUC/SP. Também é professor da Escola Superior de Propaganda e Marketing - ESPM/SP, onde também é doutorando no programa de mestrado e doutorado em negócios internacionais da ESPM/SP.E-mail: csaito@espm.br

- Vivian Iara Strehlau é Doutora pela Escola de Administração de Empresas da Fundação Getúlio Vargas - EAESP/FGV/SP. Também é Professora titular dos programas de Mestrado e Doutorado da Escola Superior de Propaganda e Marketing - PMDGI/ESPM, e coordenadora do Mestrado Profissional em Comportamento do consumidor MPCC/ESPM .E-mail: vstrehlau@espm.br 


\title{
Tourist destination choice: A bibliometric study
}

\author{
Claudio Sunao Saito and Vivian lara Strehlau
}

Escola Superior de Propaganda e Marketing - ESPM, São Paulo, SP, Brazil

\begin{tabular}{l}
\hline ARTICLE DETAILS \\
\hline Article history: \\
Received: December 12, 2016 \\
Reviewed: June 26, 2017 \\
Accepted October 06, 2017 \\
Available online November 12, 2017 \\
Double Blind Review System \\
Scientifc Editor \\
Renata galhone \\
\hline Keywords: \\
Tourism; \\
Destination Choice; \\
Decision-making process; \\
Bibliometrics; \\
Citation analysis \\
\hline
\end{tabular}

\begin{abstract}
This bibliometric study aims to understand the main subjects, approaches, and theoretical references related to travel destination choice. The Scopus database, statistical software, and citation analysis defined the relevance and prestige of the articles, authors, models, and theoretical foundations, based on the number of citations in later articles. The results demonstrate that there are a diversity of perspectives and approaches related to the topic and the articles can be grouped into studies related to decision models or destination choice, motivating factors for tourism, personal characteristics or factors, destination characteristics and attractiveness, scope of the intended trip (holiday, size, distance, duration of the trip, etc.), travel experiences (humor, feelings during the trip, post-purchase evaluations, etc.), influence of the destination's image, and influence of information/communication on the travel destination choice. The results also indicate a difference regarding the origin of the articles (countries/institution), authors, and theoretical references used in research related to the topic.
\end{abstract}

(C) 2018 Internext | ESPM. All rights reserved!

Para citar este artigo:

Saito C. S.; Strehlau V. I. (2018) Escolha de destino turístico: Estudo bibliométrico com análise de citação e co-citação de autores. Internext - Revista Eletrônica de Negócios Internacionais, 13 (1), 17-31. DOI:

http://dx.doi.org/10.18568/1980-4865.13117-31

Para acessar este artigo: http://dx.doi.org/10.18568/1980-4865.13117-31 